\title{
A new application of ultrasound-magnetic resonance multimodal fusion virtual navigation in glioma surgery
}

\author{
Chaofeng Liang ${ }^{1 \#}$, Manting Li $^{1 \#}$, Jin Gong ${ }^{11}$, Baoyu Zhang ${ }^{1}$, Cong Lin ${ }^{1}$, Haiyong He ${ }^{1}$, Ke Zhang ${ }^{2}$, \\ Ying Guo ${ }^{1}$
}

${ }^{1}$ Department of Neurosurgery, ${ }^{2}$ Department of Radiology, 3rd Affiliated Hospital of Sun Yat-sen University, Sun Yat-sen University, Guangzhou 510630, China

Contributions: (I) Conception and design: C Liang; (II) Administrative support: C Liang; (III) Provision of study materials or patients: M Li, J Gong; (IV) Collection and assembly of data: B Zhang, C Lin; (V) Data analysis and interpretation: H He, K Zhang; (VI) Manuscript writing: All authors; (VII) Final approval of manuscript: All authors.

"These authors contributed equally to this work.

Correspondence to: Ying Guo. Department of Neurosurgery, 3rd Affiliated Hospital of Sun Yat-sen University, 600 Tianhe Road, Tianhe District, Guangzhou 510630, China. Email: guoy@mail.sysu.edu.cn.

Background: Long-term survival and high-quality life of patients with gliomas depends on the extent of resection (EOR) and the protection of functional white matter fibers. The navigation system provides precise positioning for surgery based on preoperative magnetic resonance imaging (MRI) but the precision decreases when intraoperative brain drift occurs. Ultrasound (US) can support real-time imaging and correct brain shift. The real-time US-MRI multimodal fusion virtual navigation system (UMNS) is a new technique for glioma surgery. In order to obtain a maximum EOR and functional protection, this study aimed to explore the feasibility, efficiency, and safety of real-time UMNS for glioma surgery, and to evaluate the benefit of the new application by UMNS presetting markers between the tumor and functional white matter fiber surgery.

Methods: A retrospective analysis included 45 patients who underwent glioma surgery, 19 patients with only intraoperative US, and 26 patients with UMNS. A preoperative plan was made by $3 \mathrm{D}$-slicer software based on preoperative MRI. This was combined with a reconstruction of diffusion tensor imaging (DTI) that designed the important locations as "warning points" between functional white matter fibers and tumor. Following patient registration, markers were injected into preset "warning points" under image-guided UMNS in order to give us a warning during surgery in case of postoperative function deficits. The operating time, volumetric assessment in glioma resection, and postoperative complications were evaluated and used to compared those surgeries using intraoperative US (iUS) with those surgeries using intraoperate MRI (iMRI) navigation.

Results: A total of 45 patients underwent glioma surgery. Gross total removal (GTR) of iUS alone was achieved in 6 of 19 cases, while this was achieved in 22 of 26 cases with UMNS alone, demonstrating an improvement in rate of GTR from $31.58 \%$ to $84.62 \%$, respectively. This may be attributable to the superior US image quality provided by UMNS. In 13 of 26 cases, there was improved image quality (from poor/ moderate to moderate/good) with the aid of UMNS. In addition, the consistency of EOR of postoperative MRI evaluated by UMNS (92.31\%) was higher than when using iUS alone (42.11\%). The whole process of intraoperative scanning time and marker injection did not lead to a significant delay of the operating time compared to using iUS alone, and has been reported to be shorter than with iMRI as well. Furthermore, the percentage of postoperative morbidity in the UMNS group was lower than that in the iUS group (motor deficit: $11.54 \%$ vs. $42.11 \%$; aphasia: $\mathrm{P}=3.85 \%$ vs. $31.58 \%$, respectively).

Conclusions: Real-time UMNS is an effective, timesaving technology that offers high quality intraoperative imaging. Injection markers between functional white matter fibers and tumor by UMNS can help to obtain a maximum EOR of glioma and functional protection postoperatively. The integration of iUS into the neuronavigation system offered quick and helpful intra-operative images. 
Keywords: Fusion imaging; glioma; navigation; magnetic resonance imaging (MRI); ultrasound (US)

Submitted Oct 15, 2019. Accepted for publication Nov 14, 2019.

doi: 10.21037/atm.2019.11.113

View this article at: http://dx.doi.org/10.21037/atm.2019.11.113

\section{Introduction}

Glioma is the most common malignant tumor of the central nervous system. The preferred treatment is surgical resection. The area of resection has the most direct effect on prognosis. Therefore, it is of great significance to determine the tumor boundary, detect the area of surgical resection, and find residual tumor in real time (1). The traditional neuronavigation system is based on imageassisted lesion localization before surgery, but brain shift and brain deformation are prone to occur during surgery, which leads to deviation of the actual position of the tumor, residual tumor, recurrence, or dysfunction because of over excision.

The traditional navigation system cannot reflect realtime information of lesions during surgery. To ensure maximum extent of resection (EOR) with preservation of neurological function, high-field intraoperative magnetic resonance imaging (iMRI) combined with neuronavigation and integrated functional data (multimodal navigation) has been established as immediate quality control to increase the percentage of EOR and gross total removal (GTR). Furthermore, iMRI with an intraoperative update of anatomic and functional image data can overcome the general problem of brain shift, which is mainly caused by the tumor resection itself or the loss of cerebrospinal fluid (CSF) (2-5). However, the major drawbacks are that these techniques are quite expensive and require considerable time and space. Furthermore, it is impossible to supply continuous imaging guidance. Therefore, they still cannot be considered a true real-time intraoperative imaging system.

The real-time US (ultrasound)-MRI multimodal fusion virtual navigation system (UMNS) is an emerging technology that fuses US and MR images for real-time navigation. It uses a spatial magnetic localization method to align the US and MRI in real-time through image alignment. US and MRI are simultaneously displayed on the surgeon interface, with the excellent spatial resolution of MRI and the real-time simplicity of US. This study aimed to investigate the advantages and the disadvantage of the UMNS in glioma surgery in comparison to other intraoperative imaging tools, and evaluate the possible benefits of presetting markers between the tumor and the functional white matter fiber by UMNS.

\section{Methods}

Forty-five patients with glioma diagnosed by MRI were recruited from the Third Affiliated Hospital of Sun Yat-sen University (China) from November 2015 to August 2018. Twenty-six patients were operated on with UMNS, and 19 patients were operated on with intraoperative US (iUS) alone. There were 28 males and 17 females with a mean age of 49 [28-74] years old. The maximum diameter of the lesion was $40 \mathrm{~mm} \times 73 \mathrm{~mm}$. Pathology revealed 3 cases of ganglioglioma, 3 cases of anaplastic astrocytoma, 8 cases of pilocytic astrocytoma, 2 cases of diffuse astrocytoma, 6 cases of oligodendroglioma, and 23 cases of glioblastoma. Twenty-eight cases were left cerebral hemisphere lesions, and 17 cases were right cerebral hemisphere lesions.

\section{Instrument}

For MRI, a 3.0 T GE discovery 750w scanner and Omniscan contrast agent (General Electric Medical Group, USA) were used. For US, a system (MyLab Twice, Esaote S.p.A. Italy) equipped with Virtual Navigator (VN) (6) (MedCom, Germany) was used. The US probe used was a line array probe with a bandwidth of $3-11 \mathrm{MHz}$ (LA332, Esaote, Italy). The US contrast agent SonoVue (Bracco, Italy) SF6 was mixed with $5 \mathrm{~mL}$ saline.

\section{Operational processes}

\section{Preoperative registration and planning}

The preoperative MRI data (regularly 3DT1, T1WI enhancement, T2 Flair, MRA sequence) was imported into the navigation system, delineating the lesion boundary (or the area to be resected), and marking the safety boundary according to the white matter tracts reconstructed by 3 Dslicer software preoperatively. The reason for this 
was to enable the presetting of the puncture target as the "warning point" between the functional white matter fibers and tumor; this way, the system could reconstruct a threedimensional volume image containing the target and the tumor.

\section{Real-time US-MRI multimodal fusion virtual navigation assisted in accurate localization of tumor}

The technological parameters of UMNS have been described previously in detail (6). The following steps need to be completed before opening the dura:

(I) Fine-tuning. The landmark of anatomical structures such as vessels or ventricle is one of the options that might provide useful information for adjusting fusion. This step can facilitate a fusion of both imaging modalities (MRI and US) more accurately at any time to achieve the best effect of the two images being completely coincident (Figures 1,2).

(II) Evaluating the image quality in combination with contrast-enhanced ultrasonography (CEUS). Two $\mathrm{ml}$ of SonoVue suspension was injected into the internal jugular vein to make the lesion boundary more distinct (Figure 1B). In order to evaluate the intraoperative image display of tumor lesions, the US image quality of the lesions was classified into three categories according to the classification method reported by Solheim (8): (i) the image quality was good, and the boundary between the tumors was clear; (ii) the image quality was moderate, meaning it was not easy to distinguish the inside of the tumor or part of the boundary from the surrounding normal brain tissue; (iii) the image quality was poor, meaning the tumor echo and the surrounding brain tissue boundary were almost indistinguishable.

(III) Reevaluating the image quality after US-MRI imaging fusion. After US-MRI fusion was complete, the MR images were superimposed with the US images to reevaluate the lesion boundary (Figure 1C). The classification is the same as above.

(IV) Injecting markers into "warning points" under image-guided UMNS. In order to protect the functional white matter tracts, a needle equipped with the Vtrax was used to inject $0.2 \mathrm{~mL}$ of fluid gelatin mixed with methylene blue at the "warning point" for labeling under double guidance (the US image and the UMNS image) (Figures 1D,E,2).

\section{UMNS-assisted image-guided surgery}

Following the opening of the dura, CSF release, or tumor resection, traditional neuronavigation has the possibility to become less reliable because of brain shift. Fusion imaging can thus be more helpful. Intraoperative US was fused with the preoperative MRI using the landmarks in the method described above to display the residual tumor in UMNS. At this time, image quality evaluation was compared to that acquired by iUS alone. During surgery, if the "warning points" were found, this meant that the boundary was close (Figures 3,4). The decision of either GTR or partial removal (PR) as the surgical goal was defined preoperatively under special consideration of eloquent brain areas and functional MRI. At last, the surgeon evaluated the EOR through the UMNS and compared it with the postoperative MRI (Figure 5).

\section{Safety assessment}

EOR was analyzed finally by postoperative MRI which was performed within 72 hours after operation. Postoperative complications/functional improvements were observed within 2 weeks after operation, and differences were examined between UMNS patients and iUS patients.

\section{Statistical analysis}

Statistical analysis was performed using SPSS 13.0. Data were expressed as mean (minimum, maximum); differences between the two groups were analyzed by $t$-test or chisquare test, and $\mathrm{P}<0.05$ was considered statistically significant.

\section{Results}

The UMNS worked properly in all 26 patients undergoing from glioma surgery. Intraoperative US and US-MRI fusion was convenient and afforded true real-time imaging, which was valuable for evaluating the precise tumor location and the anatomical relationship between the tumor and surrounding structures (Figure 6). Neuronavigation was administered successfully with good accuracy in all cases. The mean registration error was $1.55 \pm 0.62 \mathrm{~mm}$.

\section{Extra operating time}

The total time for the UNMS process in this study was collected (Table 1). The whole process of injecting markers and scanning time with UMNS did not lead to a significant 

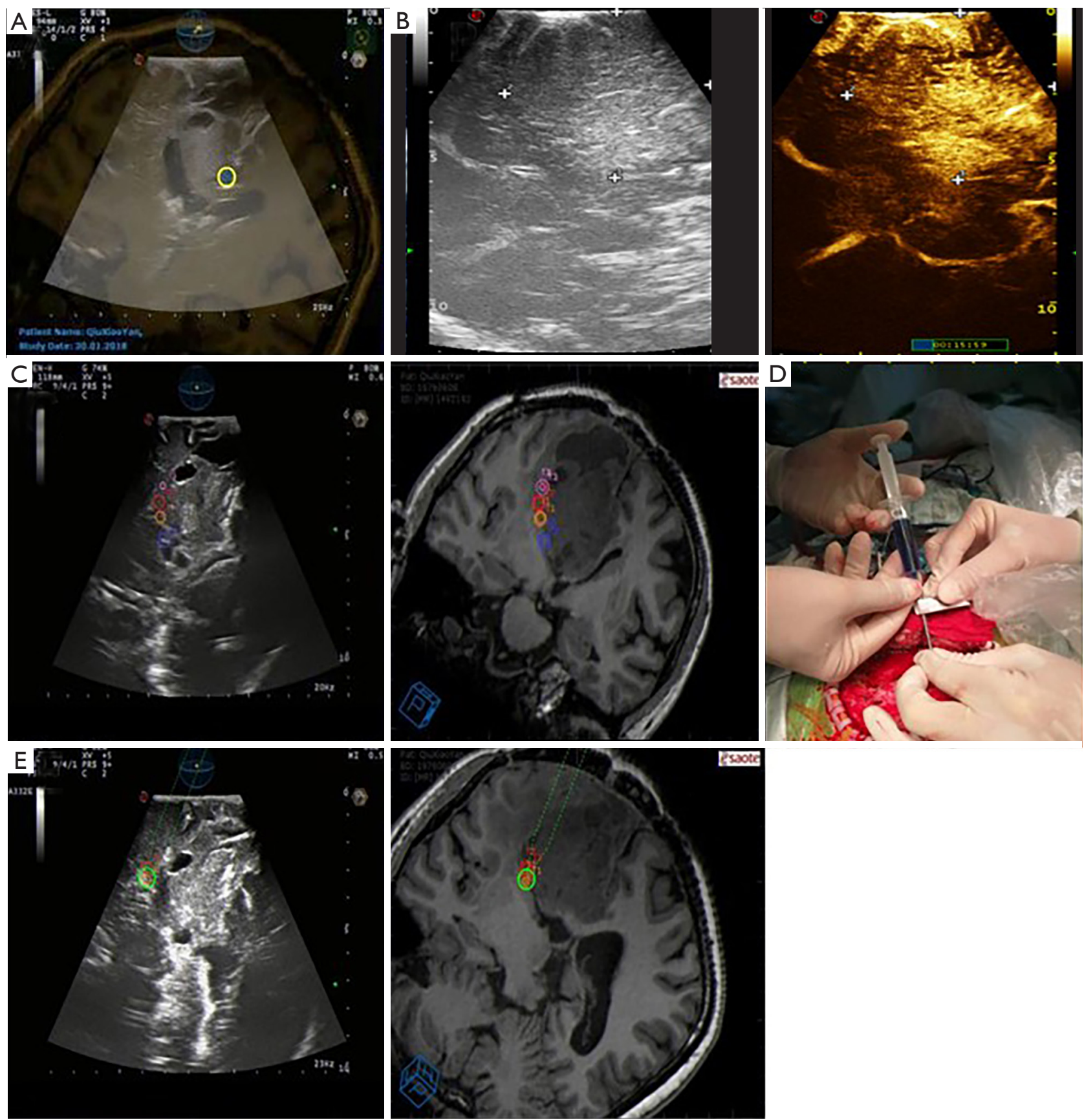

Figure 1 (A) Fine-tuning before dural opening; (B) tumor displayed more clearly in CEUS (right); (C) US was linked with MRI to display the tumor in the same screen; colorful circles indicate "warning points" planed by 3D slicer software; (D) puncture needle connected with navigation Vtrax and injected methylene blue; (E) the needle could reach the "warning point" under the help of real-time US-guided (left) and UMNS-guided (right) image; red circles indicate the "warning point", while green circles indicate the tip of the puncture needle in real-time US and virtual MRI in UMNS. CEUS, contrast-enhanced ultrasonography; US, ultrasound; MRI, magnetic resonance imaging; UMNS, US-MRI multimodal fusion virtual navigation system. 


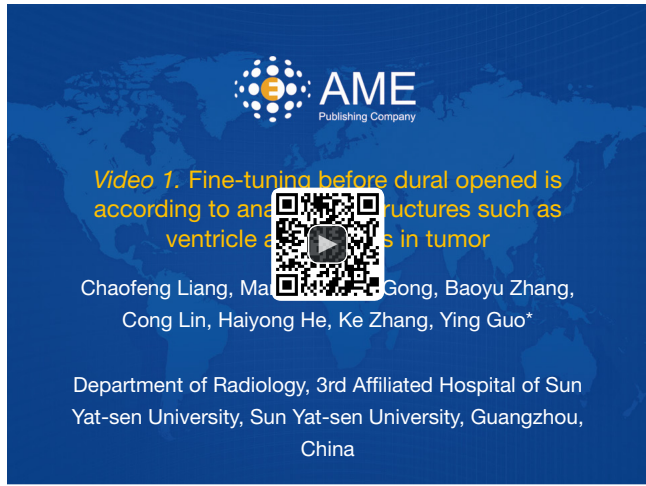

Figure 2 Fine-tuning before dural opened is according to anatomical structures such as ventricle and cavities in tumor. This step can facilitate a fusion of both imaging modalities (MRI and US) more accurately at any time to achieve the best effect of the two images being completely coincident. And then markers were injected into "warning points" under the help of real-time USguided and UMNS-guided image; red circles indicate the "warning point", while green circles indicate the tip of the puncture needle reconstructed by UMNS, and white moving point indicate the tip of the puncture needle in real-time US (7). US, ultrasound; MRI, magnetic resonance imaging.

Available online: http://www.asvide.com/watch/33028

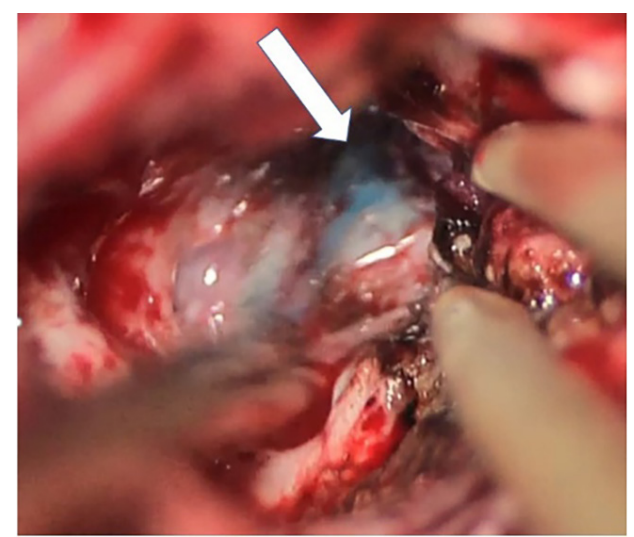

Figure 3 A "warning point" marked by fluid gelatin mixed with methylene blue (arrow); it suggested the boundary of the tumor was closed when marker was found intraoperatively.

delay in operating time compared to surgery with iUS alone.

\section{Image quality}

In our study, the image quality before dural opening had 2

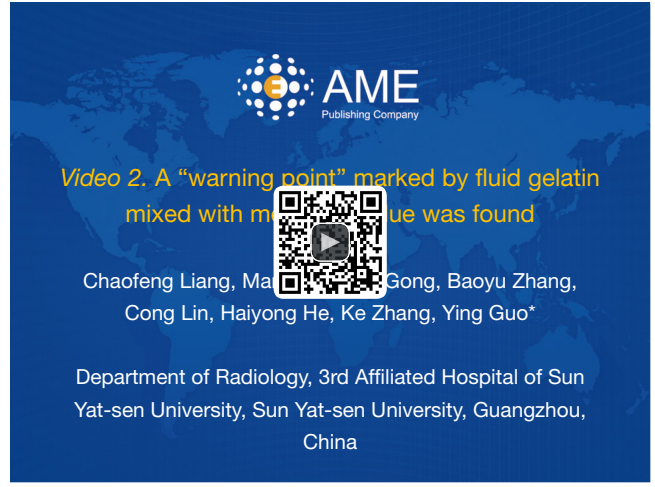

Figure 4 A "warning point" marked by fluid gelatin mixed with methylene blue was found. It suggested the boundary of the tumor was close and need to wake up the patient to monitor locomotor function in case the white matter tract occur injury (9). Available online: http://www.asvide.com/watch/33029

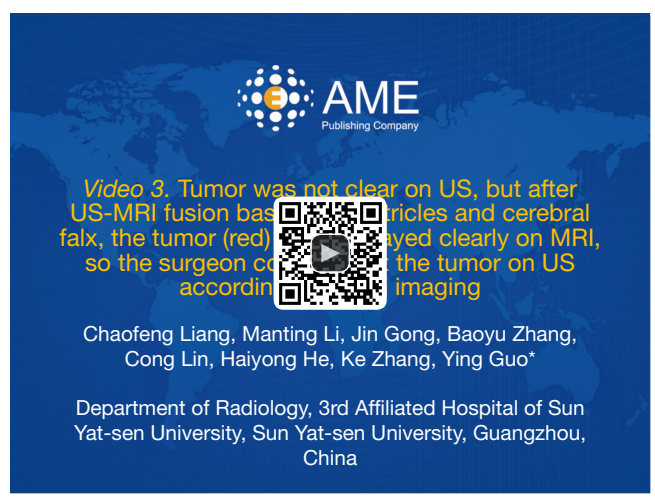

Figure 5 Tumor was not clear on US, but after US-MRI fusion based on ventricles and cerebral falx, the tumor (red) was displayed clearly on MRI, so the surgeon could detect the tumor on US according to fusion imaging; after tumor removal, real-time US fused with preoperative MRI showed a cavity in the red frame which suggested complete resection of the tumor (10). US, ultrasound; MRI, magnetic resonance imaging.

Available online: http://www.asvide.com/watch/33030

good cases, 15 moderate cases, and 9 poor cases in 26 total cases with US use alone. As for the tumor boundary, MRI showed 10 cases were clear (good), 13 cases were partly unclear (moderate), and 3 cases were unclear (poor). The image quality of 13 lesions improved (from poor/moderate to moderate/good) with the aid of UMNS. The lesion boundary of 2 recurrent glioblastomas that were poorly displayed under US were located and delineated after US- 

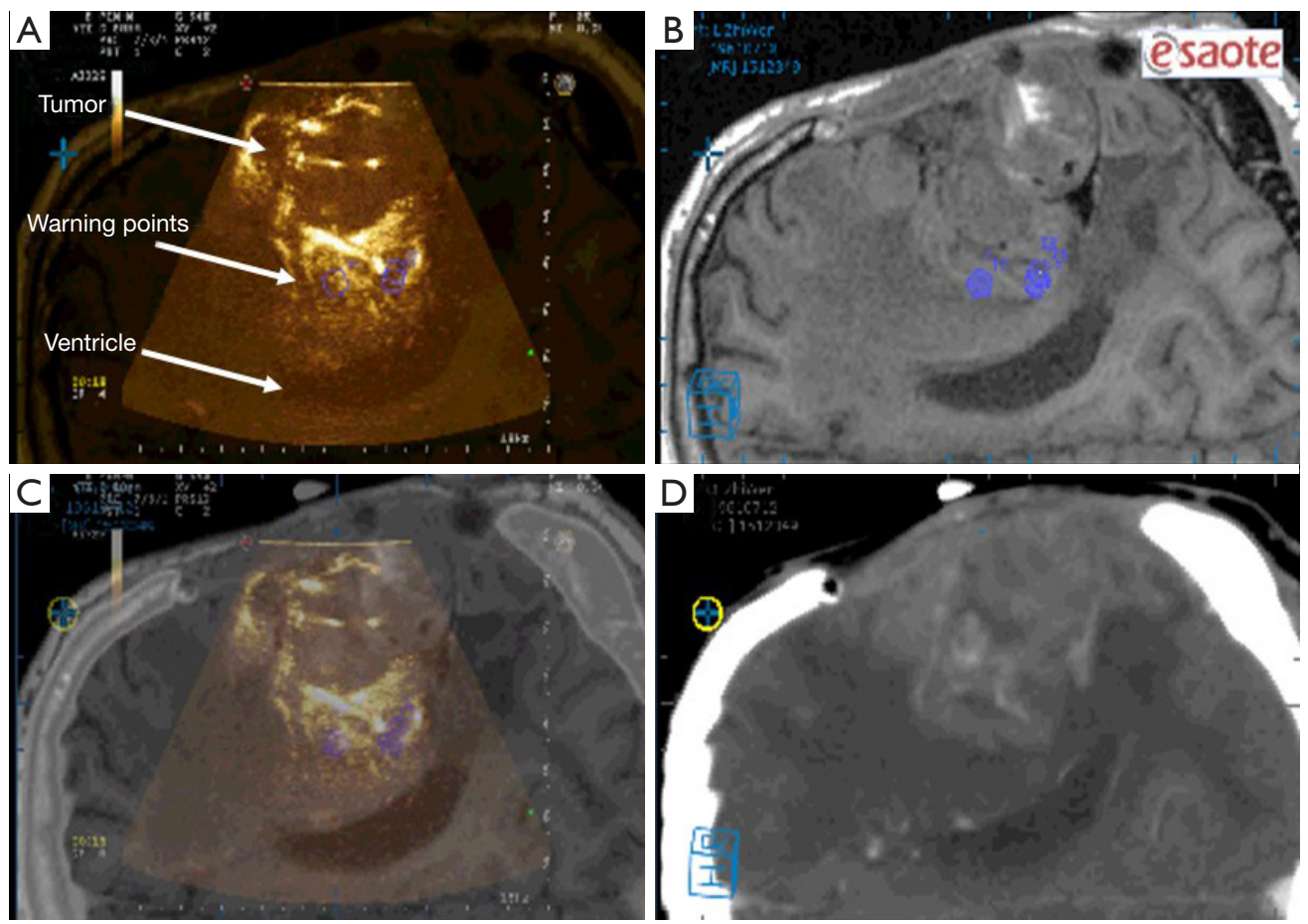

Figure 6 (A) Real-time CEUS showing the tumor, ventricle, and warning points; (B) MRI showing the virtual position in UMNS; (C) CEUS superimposed with MRI; (D) CT showing the virtual position in UMNS. CEUS, contrast-enhanced ultrasonography; MRI, magnetic resonance imaging; UMNS, US-MRI multimodal fusion virtual navigation system.

Table 1 Operating time excluding tumor removal

\begin{tabular}{lccc}
\hline Variable & Marker injection $(\mathrm{min})$ & Intraoperative scanning time $(\mathrm{min})$ & Extra operating time $(\mathrm{min})$ \\
\hline UMNS & $10.42 \pm 2.318$ & $4.38 \pm 1.329$ & $14.81 \pm 2.68$ \\
US & $10.63 \pm 1.892$ & $4.68 \pm 1.108$ & $15.32 \pm 2.19$ \\
P & 0.75 & 0.464 & 0.5 \\
\hline
\end{tabular}

US, ultrasound; UMNS, US-MRI fusion navigation system.

MRI fusion (Figure $7 A, B, C$ ). Furthermore, the maximum area of the lesion displayed on CEUS was compared with the maximum area of the lesion shown by UMNS, and the difference was significant $(\mathrm{P}<0.05)$ (Table 2).

\section{EOR}

Evaluation of the final EOR was defined by post-operative MRI. There were 24 in 26 cases $(92.31 \%)$ that showed no significant difference of EOR evaluated intraoperatively by UMNS (Figure $7 D, E$ ), while 8 in 19 cases $(42.11 \%$ ) evaluated by US alone were consistent with the results of post-operative MRI.
In our study, GTR was achieved in 6 of the 19 patients $(31.58 \%)$ when guided by intraoperative US alone, and in 22 of 26 patients $(84.62 \%)$ when guided by UMNS. Because of the infiltration of eloquent brain areas or other critical structures and in order to avoid postoperative neurological deficits, further resection was abstained from in 4 patients, and remnant tumor was still visible by UMNS at the end of operation.

\section{Postoperative deficits}

The postoperative neurological deficits were evaluated in terms motor and language ability. Using statistical analysis 

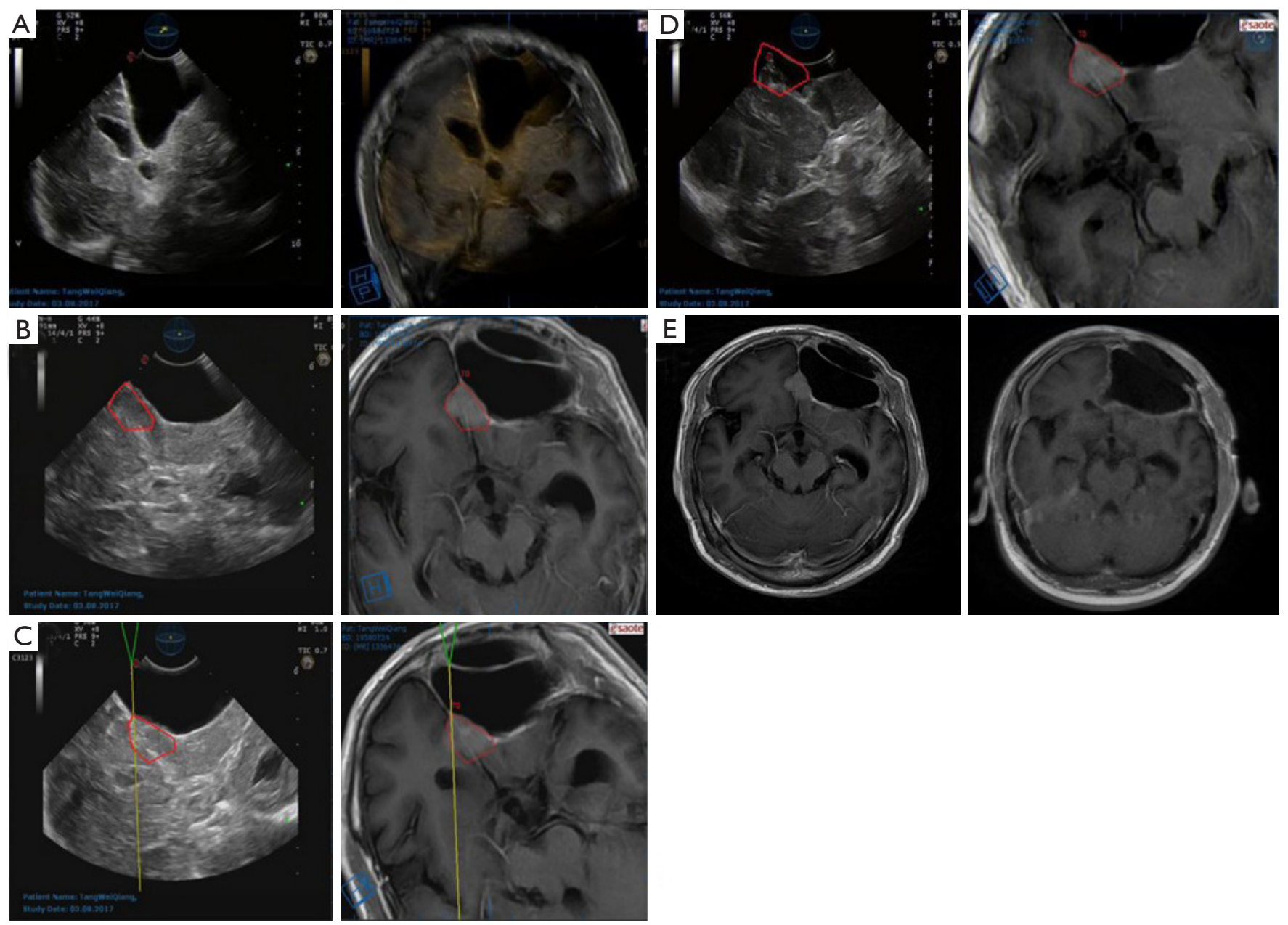

Figure 7 (A) US-MRI fusion based on ventricles and cerebral falx; (B) tumor was not clear on US, but the tumor (red) was displayed clearly on MRI, so the surgeon could detect the tumor on US according to fusion imaging; (C) Vtrax-guided resection path; (D) after tumor removal, real-time US fused with preoperative MRI showed a cavity in the red frame which suggested complete resection of the tumor; (E) pre-operative and post-operative MRI examination showed that the tumor had been removed, which was consistent with the evaluation by UMNS. US, ultrasound; MRI, magnetic resonance imaging; UMNS, US-MRI multimodal fusion virtual navigation system.

to compare the group guided by UMNS with that guided by iUS alone revealed a lower postoperative deficit in the UMNS group. Postoperative motor deficits were worse than preoperative deficits in the UMNS group, occurring in 3 of 26 patients (11.54\%) with aphasia in 1 patient $(3.85 \%)$. In contrast, 8 of 19 patients had motor deficits $(42.11 \%)$, and 6 of 19 patients $(31.58 \%)$ had aphasia in the iUS alone group. These data were collected 2 weeks after surgery.

In the UMNS group, 8 cases had stable postoperative motor deficits, and 15 cases improved after surgery. In the long-term follow-up 3 months after discharge, 7 patients $(26.92 \%)$ had further improved in comparison with the neurological status at discharge. This was considered to be caused by early postoperative edema. One patient had normal limbs after surgery, and 2 days after surgery, he underwent secondary surgery due to bleeding. No patient died, and no seizures were seen 3 months post-operation.

\section{Discussion}

Glioma is the most common malignant tumor of the central nervous system. Up to now, many studies have shown that an increasing EOR was associated with improved survival independent of age, degree of disability, WHO grade, or subsequent treatment modalities used $(11,12)$. However, it is difficult to distinguish the boundaries between glioma and 
Table 2 Summary of image quality and lesion size fused by UMNS

\begin{tabular}{|c|c|c|c|c|c|}
\hline No. & Histopathology & Image quality after UMNS fusion & US image quality & Lesion size (UMNS) (mm) & Lesion size CEUS (mm) \\
\hline 2 & Glioblastoma & Good & Moderate & $43 \times 55$ & $39 \times 41$ \\
\hline 3 & Glioblastoma & Moderate & Poor & $43 \times 62$ & $39 \times 55$ \\
\hline 4 & Glioblastoma & Poor & Poor & $45 \times 54$ & $38 \times 40$ \\
\hline 6 & Glioblastoma & Poor & Poor & $38 \times 45$ & $30 \times 37$ \\
\hline 7 & Glioblastoma & Moderate & Moderate & $33 \times 42$ & $27 \times 32$ \\
\hline 8 & Glioblastoma & Good & Moderate & $16 \times 19$ & $13 \times 18$ \\
\hline 9 & Glioblastoma & Good & Good & $42 \times 49$ & $37 \times 43$ \\
\hline 12 & Diffuse astrocytoma & Good & Poor & $29 \times 56$ & $27 \times 48$ \\
\hline 13 & Anaplastic astrocytoma & Moderate & Moderate & $40 \times 73$ & $36 \times 67$ \\
\hline 14 & Anaplastic astrocytoma & Moderate & Poor & $26 \times 33$ & $15 \times 22$ \\
\hline 15 & Pilocytic astrocytoma & Good & Good & $36 \times 37$ & $34 \times 37$ \\
\hline 16 & Glioblastoma & Poor & Poor & $36 \times 38$ & $16 \times 21$ \\
\hline 17 & Anaplastic astrocytoma & Moderate & Moderate & $31 \times 38$ & $30 \times 36$ \\
\hline 18 & Glioblastoma & Moderate & Moderate & $32 \times 48$ & $30 \times 48$ \\
\hline 19 & Ganglion cell glioma & Good & Moderate & $35 \times 46$ & $31 \times 32$ \\
\hline 25 & Glioblastoma & Good & Moderate & $26 \times 40$ & $20 \times 26$ \\
\hline 26 & Glioblastoma & Good & Moderate & $36 \times 38$ & $20 \times 21$ \\
\hline
\end{tabular}

US, ultrasound; MRI, magnetic resonance imaging; UMNS, US-MRI multimodal fusion virtual navigation system.

normal brain tissue even under microscope because of the invasive growth characteristics of glioma. Therefore, it is of great guiding significance to determine tumor boundary, detect the scope of surgical resection, and identify residual tumor in the surgery.

The traditional neuronavigation system is one of the most important auxiliary devices for neurosurgery (13). It has the advantages of high image resolution and wide field of view, and can show anatomical structures clearly.
However, the accuracy of the anatomical landmark indication is reduced if the neuronavigation system is based only on preoperative MR images. This is due to brain shift $(14,15)$ (gravity, cerebral edema, CSF release) and brain tissue deformation (brain plate, surgical operation, tissue resection and brain parenchymal expansion) (16), resulting in an actual anatomical location that does not correspond to the virtual anatomical location shown on the navigation system (17). The solution for this problem is to use 
Table 3 Study population and resection parameters of included studies

\begin{tabular}{llll}
\hline Study & N & EOR at last iMRI & Complications \\
\hline Schneider et al. [2005] (19) & 31 GBM & 11 of 31 patients GTR & One rebleed, one edema, two new paresis \\
Muragaki et al. [2006] (20) & 30 GBM & $90 \%$ EOR & NS in subpopulation \\
Nimsky et al. [2006] (21) & 57 GBM & 23 of 57 patients GTR & NS in subpopulation \\
Hatiboglu et al. [2009] (22) & 27 GBM & 24 of 27 patients GTR & NS in subpopulation \\
Lenaburg et al. [2009] (23) & 35 GBM in & 27 of 35 cases had & One respiratory failure (died), two wound infections leading to \\
& 29 patients & resection $>95 \%$ & wound revision, one CSF leak \\
Daniela, et al. [2011] (24) & 113 patients & $82.3 \%$ GTR & $7.2 \%$ motor deficits, 4.1\% aphasia \\
\hline
\end{tabular}

N, number of cases or patients; GTR, gross total resection; EOR, extent of resection; MRI, magnetic resonance imaging; NS, not specified; GBM, glioblastoma multiforme; CSF, cerebrospinal fluid.

intraoperative imaging.

Intraoperative MRI can correct brain shift and brain deformation with high-resolution images. However, it is an expensive, time-consuming, and complex operation process. More importantly, the image guidance cannot be performed continuously, so iMRI only represents a near real-time method and therefore cannot produce true real-time intraoperative images. Intraoperative US has been proven to be a valuable tool for intraoperative tumor detection (18), with the advantages of being real-time, convenient, lowcost, and non-radiative. Given the above facts, this study used a neuronavigation system to fuse real-time US with MRI, combining the high-quality image of MRI and the real-time feature of US to determine the efficiency and safety of this method in glioma surgery.

\section{Comparison of image quality with iUS}

Surgeons are more used to reading MRI than craniocerebral US imaging because of the complexity and multidimensional features of the US image. However, the boundary resolution of US for tumors is more viable than MRI. Therefore, if US could be combined with MRI, this could prove to be an optimal imaging method. The iUS image was fused with preoperative MRI by UMNS so that we could identify tumor boundaries more clearly (Figure 7B). In our study, 13 of $26(50 \%)$ lesion images had improved quality (from poor/ moderate to moderate/good) with the aid of UMNS. This improvement was mainly due to following the precisely injected "warning points" between the tumor and functional white matter fibers.

A surgeon's learning curve can also be shortened by UMNS. As intraoperative US typically has a limited field of view and low resolution, and the difference in diagnosis is highly dependent on the operator's experience, neurosurgeons need long-term training to recognize US images efficiently. Thus, the use of real-time US-MRI multimodal fusion can help neurosurgeons more easily identify anatomical structures and lesions intraoperatively, mitigating the need for extensive experience.

\section{Comparison of EOR with iMRI guiding studies}

In our study, we aimed to inject "warning points" at the important locations between functional white matter fibers and tumor, in order to remind the surgeons intraoperatively that the margin of tumor is close and caution is needed. With this new application under the aid of UMNS, our study aimed to evaluate the value of EOR and postoperative complications by UMNS with comparison to other studies of iMRI-guided resection. In these 6 selected studies, there are different results for GTR because of differing descriptions for GTR (Table 3). Two main approaches are used: qualitative (presence of contrast enhancement on T1-weighted MRI) or quantitative (volumetric assessment by manual segmentation). The first approach is a rough method with GTR assessment consisting of "yes" or "no" evaluations. The second method has the theoretical advantage of higher precision, but has unacceptably low interobserver agreement for postoperative images (intraclass correlation coefficient $=0.54$ ) to be a valid method to assess EOR. In our opinion, the former method maybe more convenient in facilitating a comparison of results between studies.

Hatiboglu reported a higher GTR rate of $66 \%$. However, this is mostly attributable to the patients with 
Table 4 Intraoperative MRI parameters of included studies

\begin{tabular}{lccc}
\hline Study & Scanning time & iMRI sequences used & Extra OR time \\
\hline Knauth et al. [1999] (25) & NS & T1 & 1 h for scanning and setup \\
Bohinski et al. [2001] (26) & Mean total 16 min & T1, T2 & NS \\
Nimsky et al. [2006] (27) & Circa 73 min per procedure & T1, T2, FLAIR, other & NS \\
Lenaburg et al. [2009] (23) & 13.5 min average & T1, T2 & NS \\
Busse et al. [2006] (28) & NS & T1, other & 13 min for advanced navigation \\
\hline
\end{tabular}

MRI, magnetic resonance imaging.

contrast-enhancing tumors in whom a $77 \%$ GTR rate can be achieved. For non-enhancing tumors, the final percentage of GTR was only $29 \%$. Our final GTR rate was comparatively high at $84.62 \%$. The possible reasons for this are outlined below.

First, a high-quality intraoperative image may contribute to a higher GTR rate. Real-time US-MRI multimodal fusion helps surgeons more accurately recognize the tumor. Virtual navigation not only helped asses the glioma features, but the fusion of preoperative MRI and real-time iUS could be displayed on the same screen, allowing the two image modes to be directly superimposed for comparison. It was more convenient and easier for surgeons to assess residual tumors through the above comparison, which combined the real-time nature of US and the high-resolution MRI from navigation to excellent advantage.

Secondly, preset "warning points" maybe help to reduce brain shift errors. Glioma grows with unclear borders, and, although the traditional neuronavigation system does provide guidance in defining the boundary based on preoperative image, brain shift and brain deformation can lead to the intraoperative deviation of the actual position of the tumor because of the tumor resection itself or the loss of CSF $(2,5)$. In our study, we injected several "warning points" at the location we planned to remove before opening the dura, in order to judge the residual tumor by distance from these points under realtime iUS and define the glioma boundary more efficiently by MRI from UMNS.

\section{Diagnostic efficacy}

The diagnostic efficacy was evaluated by the consistency of the EOR at the end of the operation as judged by surgeons and post-operative MRI as judged by radiologists. The consistency of EOR evaluated by UMNS (92.31\%) was higher than by US alone (42.11\%). The reasons for missed diagnosis maybe as follows: (I) US image quality was so low that the echo of residual tissue was close to the edema tissue around the tumor (18). The multimodality image fusion virtual navigation technology of UMNS could markedly improve the image quality. (II) The operator's experience varied. The scanning was incomplete or of the corresponding relationship between the angle of the slices and the anatomical orientation of brain was misjudged. The use of computerized techniques to merge US and MRI may overcome these drawbacks. After the images were overlapped, complementary information could have been obtained to compensate for the limitations of US.

\section{Extra operating time}

Convenience is the most obvious advantage of iUS. In this study, difference in intraoperative scanning time was not significant between the UMNS $(4.38 \pm 1.329 \mathrm{~min})$ group and the US group $(4.68 \pm 1.32 \mathrm{~min})$. However, in comparison with several studies that have shown the extra operating time of iMRI in glioma surgery (Table 4), this suggests a less time-consuming process of UMNS on average. Typically, each sequence of iMRI needs 4 to 6 minutes to acquire. In addition, iMRI requires a series of steps such as moving the operating bed and reimporting the information into the navigation system.

\section{Complications}

Since glioma grows with unclear borders and is close to the functional white matter fibers, presetting "warning points" at critical locations will help surgeons to distinguish the glioma boundary. Traditional navigation can also help to preset "warning points", but when brain shift occurs intraoperatively, these points will shift in turn. It is difficult to determine the residual tumor around the "warning point" using preoperative MRI data, so we need to update the 
intraoperative imaging into the navigation system to rectify the actual location of the tumor. The accuracy of iMRI is the highest so far, while in our study, with the help of iUS and the fusion of preoperative MRI, UMNS also greatly improved the US image quality intraoperatively, permitting the surgeon to confirm the location of the residual tumor with reference to the "warning points" and improve the EOR.

Furthermore, although "warning points" can be preset by traditional US, its accuracy is insufficient, and it is difficult to identify the location planned on preoperative MRI. Therefore, the labeled "warning points" have limited effect on avoiding the damage of functional white matter fibers. In this study, the EOR of the US group was not as good as that of the UMNS group, while the incidence of postoperative neurological deficit was higher than that of the UMNS group.

In summary, the combination of iUS and navigation can fully exploit their respective advantages. First, navigation is used to display and reconstruct the three-dimensional model of the brain and tumor, accurately locating the tumor and designing the best operative plan. Second, after the removal of the cranial bone flap, the registered iUS is used to detect the tumor location, boundary, and important structures. It is thus very convenient, time-saving, and can work in real-time. Third, in the process of excising the tumor, the surgeon can easily identify the complicating iUS image by the fusion image of preoperative MRI according to UMNS, and thus determine the location of the residual tumor and important functional area. At last, when the tumor is removed, UMNS can evaluate the EOR accurately. As iUS can provide information more economically and save both time and space when compared with other intraoperative imaging technology, UMNS combines the advantages of US and MRI-guided navigation and provides real-time intraoperative feedback. UMNS is both a safe and effective technique and can be used as an auxiliary tool for intracranial glioma surgery.

\section{Acknowledgments}

Funding: The present study was supported by Sun Yat-sen University Clinical Research 5010 Program (No. 2018025).

\section{Footnote}

Conflicts of Interest: The authors have no conflicts of interest to declare.
Ethical Statement: The authors are accountable for all aspects of the work in ensuring that questions related to the accuracy or integrity of any part of the work are appropriately investigated and resolved. The Ethics Committee approved the study of the Third Affiliated Hospital of Sun Yat-sen University [No. (2018)02-428-01].

\section{References}

1. Koekkoek JA, Vecht CJ. Postoperative seizure control in glioma patients with epilepsy. Transl Cancer Res 2017;6:S313-6.

2. Nimsky C, Ganslandt O, Fahlbusch R. Comparing 0.2 tesla with 1.5 tesla intraoperative magnetic resonance imaging analysis of setup, workflow, and efficiency. Acad Radiol 2005;12:1065-79.

3. Hall WA, Haiying L, Martin AJ, et al. Safety, Efficacy, and Functionality of High-field Strength Interventional Magnetic Resonance Imaging for Neurosurgery. Neurosurgery 2000;46:632-41.

4. Blionas A, Giakoumettis D, Klonou A, et al. Paediatric gliomas: diagnosis, molecular biology and management. Ann Transl Med 2018;6:251.

5. Hall WA, Liu H, Martin AJ, et al. Safety, efficacy, and functionality of high-field strength interventional magnetic resonance imaging for neurosurgery. Neurosurgery 2000;46:632-41; discussion 641-2.

6. Prada F, Del BM, Mattei L, et al. Preoperative magnetic resonance and intraoperative ultrasound fusion imaging for real-time neuronavigation in brain tumor surgery. Ultraschall Med 2015;36:174-86.

7. Liang C, Li M, Gong J, et al. Fine-tuning before dural opened is according to anatomical structures such as ventricle and cavities in tumor. Asvide 2019;6:343. Available online: http://www.asvide.com/watch/33028

8. Solheim O, Selbekk T, Jakola AS, et al. Ultrasound-guided operations in unselected high-grade gliomas-overall results, impact of image quality and patient selection. Acta Neurochirurgica 2010;152:1873-86.

9. Liang C, Li M, Gong J, et al. A "warning point" marked by fluid gelatin mixed with methylene blue was found. Asvide 2019;6:344. Available online: http://www.asvide. $\mathrm{com} / \mathrm{watch} / 33029$

10. Liang C, Li M, Gong J, et al. Tumor was not clear on US, but after US-MRI fusion based on ventricles and cerebral falx, the tumor (red) was displayed clearly on MRI, so the surgeon could detect the tumor on US according to fusion imaging. Asvide 2019;6:345. Available online: http://www. 
asvide.com/watch/33030

11. McGirt MJ, Chaichana KL, Gathinji M, et al. Independent association of extent of resection with survival in patients with malignant brain astrocytoma. J Neurosurg 2009; 110:156-62.

12. Tang H, Xu F, Lin L, et al. Intra-operative motor function preservation for resection of primary motor cortex meningioma. Transl Cancer Res 2018;7:1666-74.

13. Maesawa S, Fujii M, Nakahara N, et al. Clinical Indications for High-Field 1.5 T Intraoperative Magnetic Resonance Imaging and Neuro-navigation for Neurosurgical Procedures. Neurologia Medico-chirurgica 2009;49:340-9.

14. Spetzger U, Hubbe U, Struffert T, et al. Error analysis in cranial neuronavigation. Minim Invasive Neurosurg 2002;45:6-10.

15. Nimsky C, Ganslandt O, Cerny S, et al. Quantification of, visualization of, and compensation for brain shift using intraoperative magnetic resonance imaging. Neurosurgery 2000;47:1070-9.

16. Gerard IJ, Kersten-Oertel M, Petrecca K, et al. Brain shift in neuronavigation of brain tumors: A review. Med Image Anal 2017;35:403-20.

17. Stieglitz LH, Fichtner J, Andres R, et al. The silent loss of neuronavigation accuracy: a systematic retrospective analysis of factors influencing the mismatch of frameless stereotactic systems in cranial neurosurgery. Neurosurgery 2013;72:796-807.

18. Gerganov VM, Samii A, Akbarian A, et al. Reliability of intraoperative high-resolution 2D ultrasound as an alternative to high-field strength MR imaging for tumor resection control: a prospective comparative study. J Neurosurg 2009;111:512-9.

19. Schneider JP, Trantakis C, Rubach M, et al. Intraoperative MRI to guide the resection of primary supratentorial glioblastoma multiforme-a quantitative radiological

Cite this article as: Liang $\mathrm{C}, \mathrm{Li}$ M, Gong J, Zhang B, Lin C, He H, Zhang K, Guo Y. A new application of ultrasoundmagnetic resonance multimodal fusion virtual navigation in glioma surgery. Ann Transl Med 2019;7(23):736. doi: 10.21037/ atm.2019.11.113 analysis. Neuroradiology 2005;47:489-500.

20. Muragaki Y. Usefulness of intraoperative magnetic resonance imaging for glioma surgery. Acta Neurochir Suppl 2006;98:67-75.

21. Nimsky C, Ganslandt O, Buchfelder M, et al. Intraoperative visualization for resection of gliomas: the role of functional neuronavigation and intraoperative 1.5 T MRI. Neurol Res 2006;28:482-7.

22. Hatiboglu MA, Weinberg JS, Suki D, et al. Impact of intraoperative high-field magnetic resonance imaging guidance on glioma surgery: A prospective volumetric analysis. Neurosurgery 2009;64:1073-81; discussion 1081.

23. Lenaburg HJ, Inkabi KE, Vitaz TW. The Use of Intraoperative MRI for the Treatment of Glioblastoma Multiforme. Technol Cancer Res Treat 2009;8:159-62.

24. Kuhnt D, Ganslandt O, Schlaffer SM, et al. Quantification of Glioma Removal by Intraoperative High-Field Magnetic Resonance Imaging: An Update. Neurosurgery 2011;69:852-62; discussion 862-3.

25. Knauth M, Wirtz CR, Tronnier VM, et al. Intraoperative MR Imaging Increases the Extent of Tumor Resection in Patients with High-Grade Gliomas. Am J Neuroradiol 1999;20:1642-6.

26. Bohinski RJ, Warnick RE, Gaskill-Shipley MF, et al. Intraoperative Magnetic Resonance Imaging to Determine the Extent of Resection of Pituitary Macroadenomas during Transsphenoidal Microsurgery. Neurosurgery 2001;49:1133-43.

27. Nimsky C, Keller BV, Kreutzer J, et al. Intraoperative high-field magnetic resonance imaging in transsphenoidal surgery for hormonally inactive pituitary adenomas. Exp Clin Endocrinol Diabet 2007;59:105-40.

28. Busse H, Schmitgen A, Trantakis C, et al. Advanced approach for intraoperative MRI guidance and potential benefit for neurosurgical applications. J Magn Reson Imaging 2006;24:140-51. 